\title{
SUPERCONDUCTING MAGNET NEEDS FOR THE ILC*
}

\author{
J. C. Tompkins", Vl. Kashikhin (Fermilab, Batavia, Illinois), B. Parker (BNL, Upton, Long Island, \\ New York), M. A. Palmer (CLASSE, Ithaca), J. A. Clarke (ASTeC Daresbury Laboratory, \\ Warrington, UK)
}

\begin{abstract}
The ILC Reference Design Report was completed early in February 2007. The Magnet Systems Group was formed to translate magnetic field requirements into magnet designs and cost estimates for the Reference Design. As presently configured, the ILC will have more than 13,000 magnetic elements of which more than 2300 will be based on superconducting technology. This paper will describe the major superconducting magnet needs for the ILC as presently determined by the Area Systems Groups, responsible for beam line design, working with the Magnet Systems Group. The superconducting magnet components include Main Linac quadrupoles, Positron Source undulators, Damping Ring wigglers, a complex array of Final Focus superconducting elements in the Beam Delivery System, and large superconducting solenoids in the $\mathrm{e}^{+}$and $\mathrm{e}^{-}$Sources, and the Ring to Main Linac lines.
\end{abstract}

\section{INTRODUCTION}

Work on the ILC Reference Design Report [1] (RDR) began in January 2006 with the formation of an RDR structure to carry out the effort. The RDR structure included three main components: Area Systems, Technical Systems, and Global Systems. The Area Systems Groups represent the major accelerator beamline functions: e- and e+ Sources; Damping Rings; Ring to Main Linac (RTML); Main Linac; and the Beam Delivery System (BDS). The Technical Systems Groups included Vacuum Systems; Magnet Systems; Cryomodule; Cavity Package; RF Power; Instrumentation; Dumps and
Collimators; and Accelerator Physics. Finally, Global Systems Groups was composed of Commissioning, Operations \& Reliability; Control System; Cryogenics; Conventional Facilities and Siting; and Installation. A schematic view of the ILC is shown in Fig. 1 with the major components labeled by Area System.

For magnets, requirements for beam line elements were developed by the Area Systems groups; lists of magnet strengths, apertures, allowed lengths ("slot length"), field quality, and other specifications were provided to the Magnet Systems Group. The Magnet Group reviewed the requirements and worked with the Area Systems in revising the requirements as needed. Magnetic elements are specified based on the requirements at specific lattice locations, and due to the complexities of the lattice, this can result in a sizeable number of different magnet types. The Magnet Group reviewed the individual magnet specifications and wherever possible, proposed a common magnet "style" which would satisfy the requirements for several of the individual magnets and reduce the total number of magnets to be designed.

For most of the machine, certainly those elements after the Damping Rings, the beam sizes are very small and hence the magnet field quality requirements are not particularly challenging. (The Damping Rings field qualities are 'typical' storage ring requirements: field uniformities of a few parts in $10^{-4}$ of the central field are necessary.) However, there are several other very challenging requirements for the magnets to meet for the ILC to be successful.

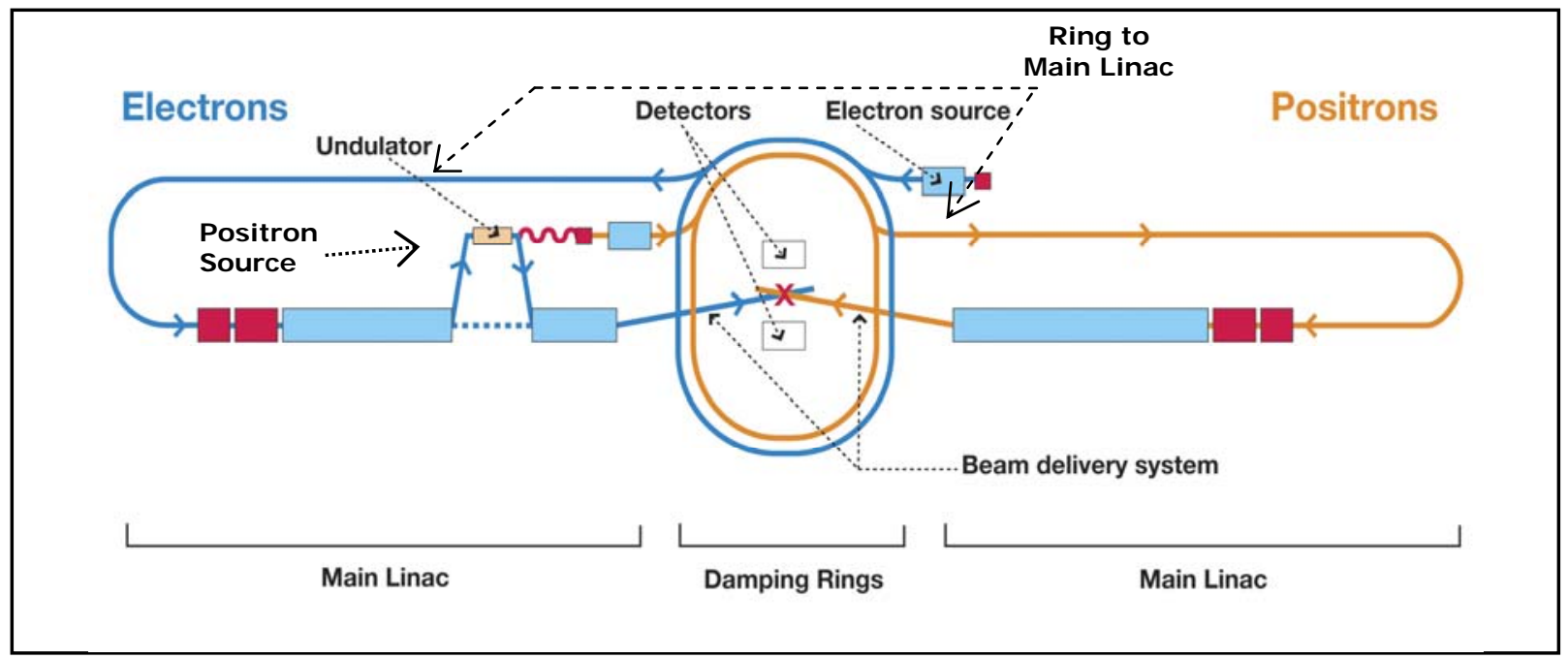

Figure 1: Schematic view of the ILC with major components identified by Area System names.

*Work supported by the U. S. Department of Energy, U. S. National Science Foundation, and the U.K. Science \& Technology Facilities Council. \#jct@fnal.gov 
The major requirements which the magnets must meet are: alignment, mechanical and magnetic stability and reproducibility, reliability, stray field limits for magnets near superconducting RF cavities, and reasonable cost.

Alignment. To maintain the extremely small beam sizes ( $\sim 6 \mathrm{~nm} \times \sim 600 \mathrm{~nm}$, nominal) required for collision, active correction is required for the beam at the quadrupoles. High precision beam position monitors (BPM's) will be mounted with each quadrupole and beam-based alignment schemes [2] will determine the beam position with respect to the quadrupole center. In the BDS, offsets will be corrected by high precision mechanical movers on which the quadrupoles are mounted; in the other areas, horizontal and vertical dipole correctors mounted with the quadrupole will be used to move the beam.

Stability and Reproducibility. The center of quadrupoles and higher order elements must remain stable over time and with changes in magnet strength. The field obtained at a given current must also be stable with respect to the magnet powering history. These requirements translate into mechanical stability in all magnets, controlling hysteretic behavior in the magnet steel, and magnetization current effects in superconducting magnets.

Reliability. A mean time between failures (MTBF) of the order of $>10^{7}$ hours [3] is required for individual magnets to meet the overall availability assigned. To achieve this value for MTBF, every aspect of magnet design, fabrication and operation will be scrutinized in a series of FMEA (Failure Modes and Effect Analysis) studies for a few representative magnets.
Stray Field, For magnets adjacent to superconducting radio frequency (SCRF) cavities, there are stringent limits on the field strength at the surface of the cavity. The present requirements are $<10 \mu \mathrm{T}$ when the cavities are cold, and $<1 \mu \mathrm{T}$ when warm [4], to prevent degradation of cavity performance.

Cost. The magnet designs must meet all specifications while minimizing the total cost of the magnet system. A balance between design, fabrication, and installation costs with those of operation must be determined.

These requirements were important considerations in developing the design approach and cost estimates for the RDR. A summary of the ILC magnet components for the $\mathrm{RDR}$ is given in Table 1 . In the RDR configuration, there are more than 13000 magnets in total, of which approximately 2300 are superconducting. (Note: individually powered coils in a single magnet assembly have been counted as separate magnets. For example, the superconducting dipole correctors in the Main Linac have both vertical and horizontal steering coils and thus are counted as two magnets.)

Due to the additional cost and complexity of most superconducting magnets, a significant effort is already underway in various $R \& D$ programs to develop the first prototypes or proof of principle for several of the major superconducting magnet systems. These will be discussed in the sections which follow.

\section{ILC SUPERCONDUCTING MAGNETS}

There are superconducting magnets in each of the ILC machine areas. In this section, we discuss the major superconducting magnet systems required, their specifications, and a brief discussion of present status.

Table 1: ILC RDR Magnet Summary Table (250GeV X 250GeV - 14 December 2006)

\begin{tabular}{|c|c|c|c|c|c|c|c|c|c|}
\hline \multirow[t]{2}{*}{ Magnet Type } & \multicolumn{2}{|c|}{ Grand Totals } & \multicolumn{2}{|c|}{ Sources } & \multicolumn{2}{|c|}{$\begin{array}{c}\text { Damping } \\
\text { Rings }\end{array}$} & \multirow{2}{*}{$\begin{array}{c}2 \\
\text { RTML } \\
\text { Qty }\end{array}$} & \multirow{2}{*}{$\begin{array}{c}2 \\
\text { Linacs } \\
\text { Qty }\end{array}$} & \multirow{2}{*}{$\begin{array}{c}2 \\
\text { BeamDel } \\
\text { Qty }\end{array}$} \\
\hline & Styles & Quantity & e- & e+ & e- & e+ & & & \\
\hline Dipole & 22 & 1356 & 25 & 157 & 134 & 134 & 716 & $\mathbf{0}$ & 190 \\
\hline Normal Cond Quad & 37 & 4182 & 93 & 871 & 823 & 823 & 1368 & $\mathbf{0}$ & 204 \\
\hline Sextupole & 7 & 1050 & $\mathbf{0}$ & 32 & 504 & 504 & $\mathbf{0}$ & $\mathbf{0}$ & 10 \\
\hline Normal Cond Solenoid & 3 & 50 & 12 & 38 & $\mathbf{0}$ & $\mathbf{0}$ & $\mathbf{0}$ & $\mathbf{0}$ & $\mathbf{0}$ \\
\hline Normal Cond Corrector & 9 & 4047 & $\mathbf{0}$ & 871 & 540 & 540 & 2032 & $\mathbf{0}$ & 64 \\
\hline Pulsed/Kickers/Septa & 11 & 227 & $\mathbf{0}$ & 19 & 46 & 46 & 52 & $\mathbf{0}$ & 64 \\
\hline NC Octupole/Muon Spoilers & 3 & 8 & $\mathbf{0}$ & $\mathbf{0}$ & $\mathbf{0}$ & $\mathbf{0}$ & $\mathbf{0}$ & $\mathbf{0}$ & 8 \\
\hline Room Temp. Magnets & 92 & 10920 & 130 & 1988 & 2047 & 2047 & 4168 & 0 & 540 \\
\hline Supercond Quad & 16 & 715 & 16 & 51 & $\mathbf{0}$ & $\mathbf{0}$ & 56 & 560 & 32 \\
\hline Supercond Sextupole & 4 & 12 & $\mathbf{0}$ & $\mathbf{0}$ & $\mathbf{0}$ & $\mathbf{0}$ & $\mathbf{0}$ & $\mathbf{0}$ & 12 \\
\hline Supercond Octupole & 3 & 14 & $\mathbf{0}$ & $\mathbf{0}$ & $\mathbf{0}$ & $\mathbf{0}$ & $\mathbf{0}$ & $\mathbf{0}$ & 14 \\
\hline Supercond Corrector & 14 & 1374 & 32 & 102 & $\mathbf{0}$ & $\mathbf{0}$ & 84 & 1120 & 36 \\
\hline Supercond Solenoid & 4 & 16 & 2 & 2 & $\mathbf{0}$ & $\mathbf{0}$ & 8 & $\mathbf{0}$ & 4 \\
\hline Supercond Wiggler & 1 & 160 & $\mathbf{0}$ & $\mathbf{0}$ & 80 & 80 & $\mathbf{0}$ & $\mathbf{0}$ & $\mathbf{0}$ \\
\hline Supercond Undulator & 1 & 42 & $\mathbf{0}$ & 42 & $\mathbf{0}$ & $\mathbf{0}$ & $\mathbf{0}$ & $\mathbf{0}$ & $\mathbf{0}$ \\
\hline Superconducting Magnets & 43 & 2333 & 50 & 197 & 80 & 80 & 148 & 1680 & 98 \\
\hline Overall Totals & 135 & 13253 & 180 & 2185 & 2127 & 2127 & 4316 & 1680 & 638 \\
\hline
\end{tabular}




\section{Main Linac Quadrupoles and Correctors}

Of the roughly 2300 superconducting magnets in the RDR, nearly 1700 are the quadrupoles and steering dipoles associated with the Main Linac cryomodulesThere are also accelerating sections in the $\mathrm{e}^{+}$and $\mathrm{e}^{-}$sources, and the RTML; the focusing quadrupoles and dipole correctors in these areas will be similar to those in the Main Linac but with strengths appropriate to lower beam energies. Table 2 lists requirements for the Main Linac quadrupole; the maximum field strength corresponds to a beam energy of $250 \mathrm{GeV}$.

Table 2: Main Linac Quadrupole Requirements.

\begin{tabular}{|l|c|}
\hline Magnetic Requirements & \\
\hline Maximum Field Strength & $54 \mathrm{~T} / \mathrm{m}$ \\
Integrated Field Strength & $36 \mathrm{~T}$ \\
& $<3 . \mathrm{e}-4$ \\
Field Non-Uniformity $\left(\mathrm{B}_{\mathrm{n}} / \mathrm{B}_{\text {fund }}\right)$ & at $\mathrm{r}_{\text {ref }}=5 \mathrm{~mm}$ \\
\hline Spatial Requirements & \\
\hline Slot Length & $660 \mathrm{~mm}$ \\
Bore diameter & $90 \mathrm{~mm}$ \\
Beam pipe & $78 \mathrm{~mm}$ \\
\hline Alignment Tolerances (installation) & \\
\hline Position (magnetic center) & $0.3 \mathrm{~mm} \mathrm{rms}$ \\
Pitch, yaw, roll (magnetic axis) & $0.3 \mathrm{mrad}$ \\
\hline Alignment Tolerances (beam based) & \\
\hline Position (magnetic center) & $\sim 1 \mu \mathrm{m}$ \\
Pitch, yaw, roll (magnetic axis) & $<0.3 \mathrm{mrad}$ \\
\hline
\end{tabular}

The cryomodule layout is based on the design of the DESY TESLA cryomodule [5]. One significant change from the earlier DESY cryomodule is the re-location of the quadrupole-corrector-BPM assembly from the end of the module to the middle where it sits under the central support post. Fig. 2 shows an early conceptual layout of the magnet package mounted under the central support.

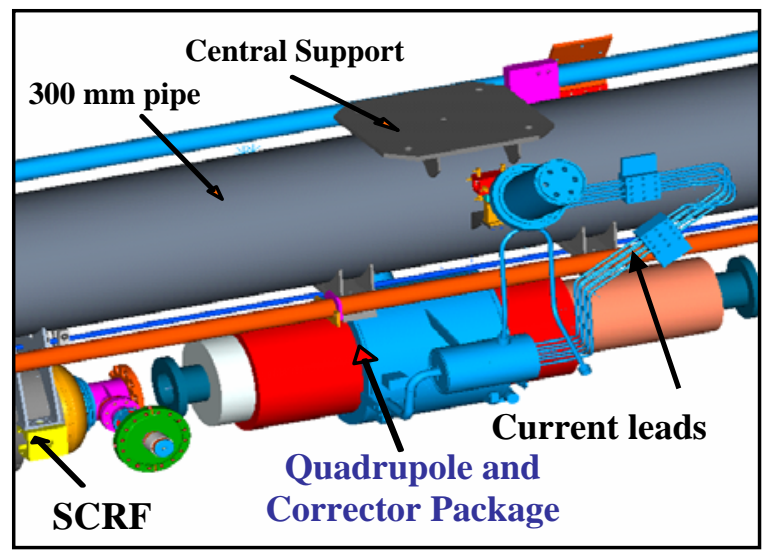

Figure 2: Conceptual layout of a quadrupole-corrector BPM assembly mounted at the center of a cryomodule.

The critical requirements for the quadrupole and corrector system are the stability and reproducibility of the magnetic center and the field gradient. The use of beam-based alignment requires that the quadrupole gradient remain independent of corrector strengths as well as controlling hysteretic behavior in the quadrupole windings.
A model of a similar quadrupole which included nested correction coils was developed by CIEMAT (Spain) for the TESLA Test Facility[6] and is soon to be the subject of a magnetic stability study at SLAC [7]. R\&D programs for ILC specific designs are presently under way at Fermilab [8] and KEK [9].

\section{Damping Ring Wigglers}

The Damping Rings play a crucial role in the ILC accelerator chain: they must accept and reduce the large emittances of the incoming $\mathrm{e}-$ and $\mathrm{e}+$ beams to required levels within the $200 \mathrm{~ms}$ interval between machine pulses. To achieve the short damping times required, roughly 200 $\mathrm{m}$ of superconducting wigglers are used in each ring. The wigglers are based on the Cornell CESR-c design [10] and operate at $4.5 \mathrm{~K}$. The design is "superferric" with the superconductor wound around the outside of the iron pole pieces (see Fig. 3). Due to the large synchrotron radiation load, the wiggler vacuum chamber is designed as a "warm bore insert" with integral cooling, which is an independent mechanical assembly. The total absorbed power per wiggler is $26 \mathrm{~kW}$.

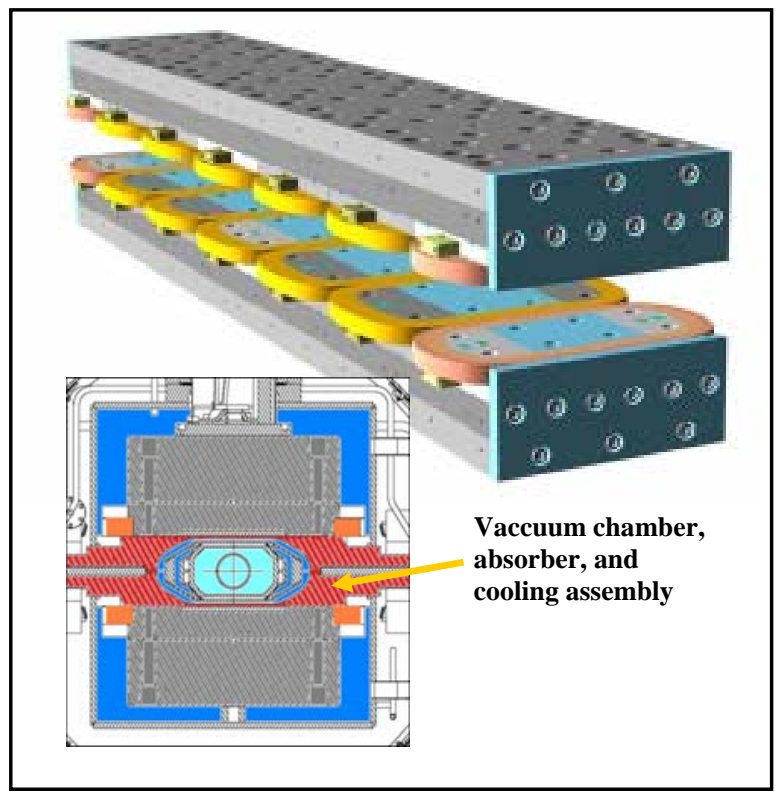

Figure 3: Modified CESR-c wiggler design; the details of the vacuum chamber, absorber, and cooling channels are visible in the insert.

The RDR wiggler design differs from the CESR-c design in length: the CESR-c design was $1.3 \mathrm{~m}$ in length, the ILC version is $2.5 \mathrm{~m}$. Other wiggler parameters were essentially the same. A total of 160 superconducting wiggler magnets (80 per ring) will be required.

Beyond the RDR design, an increase in the gap is being considered to allow more space to handle the synchrotron radiation load and design details will be reviewed to provide a simplified assembly. The parameters of an ILC optimized wiggler design are found in Table 3. 
Table 3: Parameters for an ILC Optimized CESR-c Wiggler.

\begin{tabular}{|c|c|c|}
\hline Parameter & Unit & Value \\
\hline Peak Field & $\mathrm{T}$ & 1.95 \\
\hline Number of poles & & 12 \\
\hline Total length & $\mathrm{m}$ & 1.68 \\
\hline Period & $\mathrm{m}$ & 0.32 \\
\hline PoleWidth & $\mathrm{cm}$ & 23.8 \\
\hline Gap Height & $\mathrm{cm}$ & 8.6 \\
\hline$\Delta \mathrm{B} / \mathrm{B} 0$ at $\mathrm{x}=10 \mathrm{~mm}$ & & $6.0 \times 10-4$ \\
\hline Coil Current & $\mathrm{A}$ & 141 \\
\hline Beam Energy & $\mathrm{GeV}$ & 5 \\
\hline
\end{tabular}

\section{$e^{+}$Source Undulator}

The positrons are created by pair production from an intense photon beam created by a helical undulator installed in the Main Linac electron beam at the $150 \mathrm{GeV}$ point. The helical undulator generates twice the synchrotron radiation power per period compared to a planar undulator and it also produces polarized photons which can provide longitudinally polarized positrons.

The design of the undulator presents many challenges. The short periods require high fields which pushes limits of technology. The narrow apertures require very tight tolerances to maintain alignment. The undulator will have a cold $(4 \mathrm{~K})$ bore tube: heat loads to the conductor must be kept low. The very small aperture makes the use of conventional vacuum pumps impossible. The undulator must have minimal impact on the electron beam while removing energy from the electrons.

Undulator development has been underway in Europe by the HeLiCal collaboration [11]. A double helical coil, wound on a $6.35 \mathrm{~mm}$ diameter $\mathrm{Cu}$ tube, with an inner diameter of $5.85 \mathrm{~mm}$, provides a helical transverse field. Successful tests of several prototypes have resulted in the choice for the RDR design [12]. A complete undulator module as listed in Table 4 is presently under construction in the UK and will be complete by the end of 2007.

Table 4 lists the main specifications for the undulator at the time of the RDR; a cut-away drawing of an undulator module revealing its major components is shown in Fig. 4.

Table 4: Summary of Undulator Specifications.

\begin{tabular}{|c|c|}
\hline Parameter & Value \\
\hline On axis field & $0.86 \mathrm{~T}$ \\
\hline Peak to peak variation & $<1 \%$ \\
\hline Period & $11.5 \mathrm{~mm}$ \\
\hline Nominal Current & $\sim 250 \mathrm{~A}$ (80\% of short sample) \\
\hline SC wire & $\begin{array}{c}\text { NbTi 0.4mm dia., } \\
\text { SC:Cu ratio 0.9:1 }\end{array}$ \\
\hline Winding Cross Section & 7 wires wide x 8 high \\
\hline Number per module & 2 \\
\hline $\begin{array}{c}\text { Length of magnetic } \\
\text { field }\end{array}$ & $2 \times 1.74 \mathrm{~m}$ \\
\hline Number of modules & 42 \\
\hline
\end{tabular}

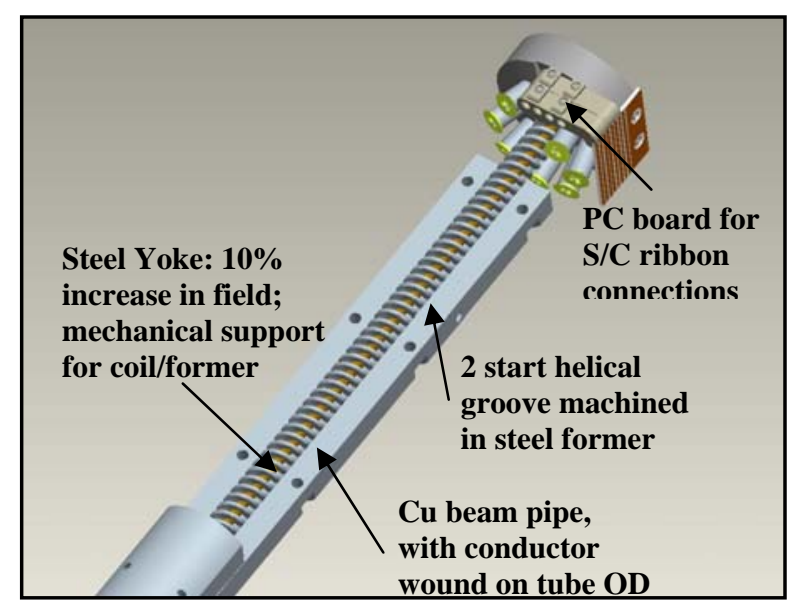

Figure 4: Details of the design of an undulator module

A similar design is being pursued at Cornell (Mikhailichenko and Tigner [13]) with a slightly larger beam tube aperture and modified coil end geometry.

\section{BDS Superconducting Magnets}

The interaction region (IR) is complicated: even in the 14 mrad crossing angle configuration (as compared to the 2 mrad option), the incoming and outgoing beams are in very close proximity. The presence of a detector with a strong solenoidal field adds further complications to the design of the beam line. In the RDR two detector "push-pull" scenario, elements of the beam line are captured with the detectors and must move with them.

The beam position must be exceptionally stable - the beam vertical width is less than $10 \mathrm{~nm}$ - for collisions to occur, which requires extremely high mechanical and magnetic stability of the final focus elements. There are significant radiation loads from interactions, "beamstrahlung" and the disrupted beams which add further design constraints.

The IR has been continuously evolving with significant changes taking place during the RDR period. Brett Parker (BNL), responsible for the design of these superconducting magnets, has been providing novel solutions to meet the many challenging constraints in this area [14]. Other superconducting magnets in the BDS include a dipole "detector integrated dipole" - located around the intersection point on the outer support cylinder of the solenoid to compensate for the vertical deflection of the incoming beam, which is at an angle to the solenoid axis; and octupole correctors - "tail-folding octupoles" - which are upstream of the IR..

Fig. 5 displays a conceptual layout of the final focus region elements as arranged in two separate cryostats in the RDR design. The designs of the individual magnet windings in these assemblies are refined as the details of the detectors and the beamlines mature. 


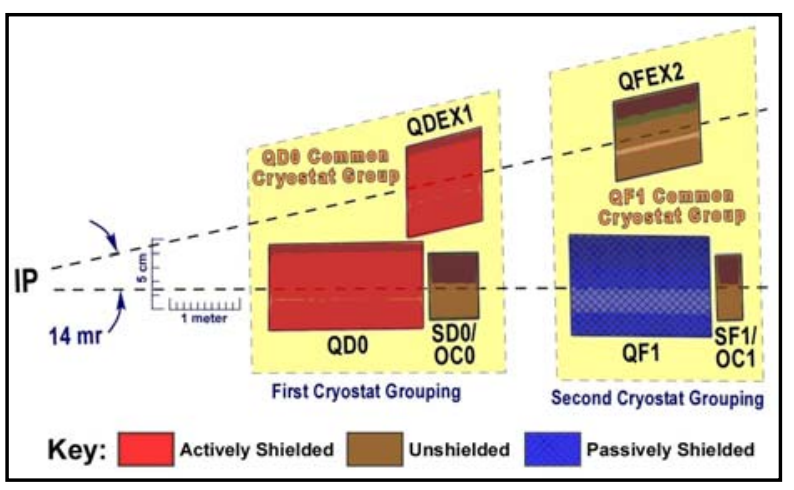

Figure 5: Conceptual layout of the IR superconducting magnet assemblies; the first group, labeled "First Cryostat Grouping”, moves with the detector

Table 5 below provides a detailed list of the IR magnets used in the RDR design and reveals more of the complexity of magnet design in this area. The magnets grouped in "QD0 Group" must move with the detectors; those in the second group, "QF1 Group", are fixed in the beam line. The magnets are also grouped according to incoming beam or disrupted beam lines; the very close spacing of the magnetic elements in the two lines requires additional shielding coils to limit flux from one line affecting the other. The magnetic elements in the two different cryostat groups are listed in Table 5 . Note that 4 QD0 Group assemblies are required (2 per detector), while only 2 QF1 Group assemblies are needed in the fixed beam lines.

Table 5: RDR SC Magnet Components at the IR.

\begin{tabular}{|c|c|c|}
\hline & QD0 Group & QF1 Group 2 \\
\hline \multirow{12}{*}{ 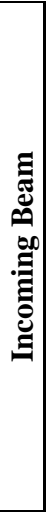 } & QD0 & QF1 \\
\hline & Main Quadrupole & Quadrupole \\
\hline & Dipole & Dipole \\
\hline & Skew Dipole & Skew Dipole \\
\hline & Skew Quadrupole & Skew Quadrupole \\
\hline & Shield Quadrupole & \\
\hline & SD0 & SF1 \\
\hline & Octupole & Octupole \\
\hline & Sextupole & Sextupole \\
\hline & Skew Sextupole & Skew Sextupole \\
\hline & Dipole & Dipole \\
\hline & Skew Dipole & Skew Dipole \\
\hline \multirow{6}{*}{ 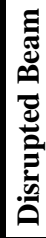 } & QDEX1 & QFEX2A \\
\hline & Main Quadrupole & Quadrupole \\
\hline & \begin{tabular}{|l|} 
Dipole \\
\end{tabular} & Dipole \\
\hline & Skew Dipole & Skew Dipole \\
\hline & Skew Quadrupole & Skew Quadrupole \\
\hline & Shield Quadrupole & \\
\hline
\end{tabular}

\section{Superconducting Solenoids}

Large aperture, high field strength solenoids are needed in several areas: e+ and e- Sources, RTML, and BDS. In the RDR, a total of 16 solenoids of 4 different styles were determined to require a superconducting design; there were 50 conventional solenoids of 3 different styles. In the short time (and limited resources) allotted for the
RDR, only rough conceptual designs were developed. More detailed designs are needed to obtain a more complete understanding of energy deposition heat loads and tradeoffs between capital and operating costs.

\section{ACKNOWLEDGEMENTS}

The authors acknowledge the many contributions of the members of the ILC RDR Magnets Systems Group: Paul Bellomo, Cherrill M. Spencer, SLAC; Eduard N. Bondarchuk, Boris Kitaev, Efremov Inst.; Jin-Young Jung, Steve Marks, Dave Plate, GianLuca Sabbi, Ross Schlueter, LBNL; Thomas Mattison, UBC, Nikolay Morozov, JINR, Ryuhei Sugahara, KEK, Mike Tartaglia, Linda Valerio, FNAL, and David Warner, CSU.

\section{REFERENCES}

[1] N. Phinney, ed., "International Linear Collider Reference Design Report 2007”, ILC-REPORT-2007001

[2] P. Tenenbaum and T. O. Raubenheimer, PRST - AB 3, 052801 (2000)

[3] T. Himel, private communication.

[4] From C. Spencer, private communication, with minor modifications for use in this paper.

[5] TESLA Technical Design Report, Part II: “The Accelerator,", edited by R. Brinkmann K. Flöttmann J. Roßbach, P. Schmüser N. Walker, and H. Weise, TESLA Report 2001-22

[6] F. Toral, et al., "Fabrication and Testing of a Combined Superconducting Magnet for the TESLA Test Facility”, Proceedings of the MT19, Sept. 2005, Genova, Italy

[7] C. Adolphsen, private communication.

[8] Vl. Kashikhin, Fermilab Internal Design Review, April 2007.

[9] N. Ohuchi, KEK, private communication.

[10] J. Urban and G. Dugan. "CESR-c Wiggler Studies in the Context of the International Linear Collider Damping Rings". In Proceedings of Particle Accelerator Conference 2005, Knoxville, TN, May 2005.

[11] http://www.astec.ac.uk/id mag/ID-Mag_Helical.htm

[12] D. J. Scott, et al., PRST - AB 10, 032401 (2007)

[13] A. Mikhailichenko, Tests of a Superconducting Undulator for ILC, EPAC 2006, p. 813

[14]B. Parker, et al., "The Superconducting Magnets of the ILC Beam Delivery System”, PAC’07, Albuquerque, June 2007,THPMS091 